\title{
PERCEPCIÓN LINGÜÍSTICA Y PLURICENTRISMO: ANÁLISIS DEL BINOMIO A LA LUZ DE LOS RESULTADOS DEL PROYECTO LINGUISTIC IDENTITY AND ATTITUDES IN SPANISH-SPEAKING LATIN AMERICA (LIAS)
}

Language perception and Pluricentrity: Analysis of the binominal in the light of the results
from the Project Linguistic Identity and Attitudes in Spanish-speaking Latin America (LIAS)

\author{
Carla Amorós-Negre \\ Universidad de Salamanca \\ carlita@usal.es \\ Miguel Ángel Quesada Pacheco \\ Universidad de Bergen \\ miguel.quesada@if.uib.no
}

Enviado: $20 / 12 / 2018$

Aceptado: 22/06/2019

\section{Resumen}

Este trabajo defiende la íntima relación existente entre conciencia sociolingüística y pluricentrismo lingüístico y propone analizar esta vinculación en profundidad, dado que la percepción de los hablantes hacia las distintas variedades y variantes lingüísticas es fundamental en la configuración y surgimiento de modelos idiomáticos. En este sentido, y a la luz de los datos aportados por el Proyecto actitudinal LIAS, Linguistic Identity and Attitudes in Spanish-speaking Latin America (Chiquito y Quesada-Pacheco eds. 2014), se estudia, con una metodología cuantitativa, el grado de aceptación y legitimación que muestran los hablantes nativos de español de diferentes proceden-

\begin{abstract}
This paper advocates the close linkage among sociolinguistic awareness and language pluricentrity and calls for a deeper exploration of this relationship, due to the fact that speakers'perceptions towards different language varieties and variants is crucial in the setting and emergence of linguistic norms. In this sense, and, in the light of the data obtained from the attitudinal Project LIAS, Linguistic Identity and Attitudes in Spanish-speaking Latin America (Chiquito \& Quesada-Pacheco eds. 2014), we study, in accordance with a quantitative methodology, the level of acceptance and legitimation that Spanish native speakers from different origins show towards the other Spanish
\end{abstract}

Para citar este artículo / To cite this article: Amorós-Negre, Carla y Miguel Ángel Quesada Pacheco (2019). Percepción lingüística y pluricentrismo: análisis del binomio a la luz de los resultados del proyecto Linguistic Identity and Attitudes in Spanish-speaking Latin America (LIAS). ELUA, 33: 9-26. doi: 10.14198/ELUA2019.33.1

Enlace / Link: http://dx.doi.org/10.14198/ELUA2019.33.1 
cias hacia el resto de variedades hispanas. Solo con una mayor atención al componente actitudinal se puede determinar el grado de ejemplaridad que los hablantes confieren a unas u otras variedades nacionales del español.

PALABRAS CLAVE: actitudes lingüísticas, folklinguistics, pluricentrismo, estandarización, variedades del español. varieties. It is only with a closer attention to the attitudinal component that it is possible to determine the degree of prestige that speakers grant to or other Spanish national varieties.

KEYWORDS: Language attitudes, Folklinguistics, Pluricentrity, Standardization, Spanish varieties.

\section{INTRODUCCIÓN}

Los estudios sobre actitudes lingüísticas han adquirido desde hace décadas un gran interés científico con aportaciones desde la psicología social, la sociolingüística, la sociología, etc. y desde orientaciones tanto conductistas como mentalistas, centradas en aspectos cognitivos, afectivos y/o conductuales (Ryan y Giles 1982; López Morales 2003 [1989]; Gimeno 1993 [1990]; Cargile et alii 1994; Alvar ed. 1996a., ed. 1996b; Albarracín et alii 2005 , etc.). Asimismo, también la antropología y la sociolingüística crítica han enfatizado la conveniencia de alejarse de posturas behavioristas o mentalistas y explorar, desde un punto de vista cualitativo, el terreno de las ideologías lingüísticas (cfr. Woolard 2008; Arnoux y Del Valle 2010, etc.). A nuestro entender, las diferentes aproximaciones pueden contribuir a ampliar el conocimiento científico sobre la reflexividad de los mismos hablantes en torno al fenómeno lingüístico. Este es, justamente, uno de los objetivos de este trabajo: atender a las repercusiones que para la ciencia lingüística como disciplina social tiene la percepción del hablante común sobre la realidad lingüística circundante, una aproximación bautizada ya en 1966 por Hoenigswald como Folk-linguistics, que sigue necesitando de muchas aportaciones para adquirir un conocimiento más integral del funcionamiento social del lenguaje.

Es evidente que todos los hablantes asignamos valor a nuestras realidades circundantes y el lenguaje y las lenguas no constituyen una excepción. Es más, toda persona al ser hablante de al menos de una lengua materna se siente legitimada para emitir juicios de valor sobre una facultad que posee desde el nacimiento y con la cual no solo se comunica sino que se identifica. El hablante posee, en efecto, un conocimiento lingüístico interiorizado implícito y procedimental distinto del conocimiento que un lingüista puede explicitar y convertir en conocimiento declarativo.

Todos los hablantes manifestamos actitudes de forma más o menos abierta o encubierta y más o menos consciente o inconsciente hacia las lenguas y sus respectivos hablantes, en forma de opiniones, censuras, críticas, etc. fruto de la percepción de usos lingüísticos propios y ajenos. A este respecto, interesa señalar que íntimamente ligada a la reflexión idiomática del hablante aparece la normatividad porque en toda comunidad lingüística se generan juicios de valor cuando se percibe la heterogeneidad dialectal. En este sentido, las actitudes lingüísticas son una muestra del comportamiento prescriptivo universal; proporcionan información de primera mano sobre los valores culturales de una comunidad de habla y tienen evidentes repercusiones en la dinámica de la variación y del cambio lingüísticos ( $c f r$. Preston 1989; Niedzielski y Preston 2000; Moreno Fernández 2012; Cestero y Paredes 2013; Caravedo 2014, etc.). Los discursos normativos son el espejo del conocimiento reflexivo intrínseco del ser humano, que se van constituyendo como producto histórico y cultural (cfr. Volóshinov 1986). 
En la actualidad, el pluricentrismo lingüístico es, precisamente, el modelo hacia el que muchas lenguas están orientando la estandarización / ejemplaridad lingüística y organizando, en consecuencia, la normatividad. En este sentido, una de las ideas centrales de la noción de pluricentrismo es la de que los hablantes de las diferentes variedades ejemplares, al reconocerles a sus usos lingüísticos un valor simbólico en la construcción de su identidad colectiva, deben tener potestad y autonomía para conseguir, sobre la base de sus modelos de uso lingüístico, una normalización y/o una estandarización propias de la variedad que hablan (Amorós-Negre y Prieto de los Mozos 2017; Villena Ponsoda y Vida Castro 2017). Los últimos estadios en la culminación de dicho proceso podrían, pues, encaminarse hacia una codificación diferencial de carácter "endonormativo", basada en la variedad ejemplar de la propia comunidad (Stewart 1968: 534), pero existen diferentes etapas hasta la consolidación de un pluricentrismo verdaderamente simétrico entre las diferentes variedades de una lengua ( $c f r$. Auer, Hinskens y Kerswill eds. 2005; Muhr 2012). En dicho proceso influyen, además de las políticas lingüísticas implementadas por las autoridades lingüísticas tradicionalmente legitimadas — academias, lingüistas, escuelas, medios de comunicación, etc. — las creencias e ideologías de los propios hablantes sobre su variedad, sobre las convergencias y divergencias que se establecen con el tradicional estándar codificado de la lengua histórica que hablan, porque también los hablantes son agentes normativos decisivos en las cuestiones concernientes a los modelos de corrección y ejemplaridad.

\section{OBJETIVOS Y METODOLOGÍA}

Son varios los investigadores que coinciden en señalar que las actitudes lingüísticas no han constituido un objeto de interés prioritario para la sociolingüística hispánica, por lo que poseemos todavía un conocimiento muy impreciso al respecto (Blas Arroyo 1999; Bugel ed. 2014; Cestero y Paredes 2015a). "Entre los estudios que hacen falta está el de los valores que dan vida a las normas de cada región o de cada país hispánico" (Lara 2004: 111), por lo que se necesita trabajar por una mayor aproximación no solo a los comportamientos sino a las actitudes lingüísticas de los hablantes, un aspecto crucial para la definición de los polémicos estándares de las lenguas y, en particular, del español.

Así pues, con el fin de acercarnos a las percepciones lingüísticas de los hablantes nativos de español en torno a sus modelos lingüísticos y contribuir a analizar hasta qué punto el pluricentrismo lingüístico, esto es, la aceptación de varias normas lingüísticas de referencia para una misma lengua, ha cristalizado entre la población hispanohablante, nos serviremos de los datos aportados por un magno proyecto de alcance panhispánico, el Linguistic Identity and Attitudes in Spanish-speaking Latin America (LIAS), que ha dado lugar a la publicación de Actitudes lingüísticas de los hispanohablantes ante el idioma español y sus variantes (Chiquito y Quesada-Pacheco eds. 2014). Se realizará, por tanto, un análisis cuantitativo y comparativo de los diversos resultados hallados en 20 capitales del mundo hispánico. Sin duda, el carácter panhispánico del proyecto, así como la sistematicidad y homogeneidad en la recogida y análisis de muestras muy uniformes y representativas de las diversas sintopías nos ha permitido hallar las tendencias actitudinales de los hablantes nativos capitalinos de veinte países hispanos en lo concerniente a la cuestión del pluricentrismo lingüístico, datos que pueden contrastarse con otros procedentes de importantes proyectos sobre estudios actitudinales que se están llevando a cabo actualmente, tales 
como el PRECAVES XXI (Proyecto para el estudio de las creencias y actitudes hacia las variedades del español en el siglo XXI) (cfr. Cestero y Paredes 2013, 2015a, 2015b y Paredes y Cestero 2018).

\section{EL PROYECTO LIAS: TENDENCIAS ACTITUDINALES EN EL MUNDO HIS- PANOHABLANTE}

El Proyecto Linguistic Identity and Attitudes in Spanish-speaking Latin America (LIAS) incluyó la realización de 8000 entrevistas en 2011 a hablantes de español como L1 — 400 informantes de las 20 capitales de la hispanofonía en las que el español es lengua originaria-, cuyos resultados fueron analizados por diferentes investigadores de las diversas regiones hispánicas. La estratificación sociolingüística de la muestra tuvo en cuenta la distribución porcentual de la población de cada nación hispanohablante según los datos censales. Para la elicitación de los datos se empleó una entrevista directa con un cuestionario en la variedad culta de la zona, compuesto por 30 preguntas cerradas y abiertas de tipo cognitivo, afectivo y pragmático. Se preguntó a los encuestados sobre su propia variedad materna (percepción tanto cognitiva como afectiva —en qué región del país se habla más parecido/más diferente; mejor/peor la variante nacional; el nombre que le otorgan a su lengua materna, etc.-). Posteriormente, se inquirió también sobre las diferentes variedades del español comparadas con la propia (valoración cognitivo-afectiva del español de otros países hispánicos, preferencias del español de los medios de comunicación, etc.), centrando la atención en dos aspectos muy presentes en la investigación actitudinal, en general, y del mundo hispánico, en particular, a saber, la corrección y la unidad de la lengua.

Ya los trabajos actitudinales pioneros del ámbito anglosajón pusieron de relieve que las valoraciones de la mayoría de hablantes se basan en apreciaciones y creencias más o menos acertadas sobre sus variedades lingüísticas, pero la mayoría se fundamenta no en cualidades lingüísticas intrínsecas sino en variables de naturaleza social, política o económica. En efecto, así se observa en los comentarios que muchos informantes del Proyecto manifestaron, por ejemplo, a propósito de la propia variedad de español o de la de otras naciones: el español que se habla en Chile dicen los mismos hablantes capitalinos "tiene palabras inventadas que no se encuentran en el diccionario" (Rojas 2014: 149); para los ecuatorianos de la capital, el habla de Cuenca, Guayaquil y Tulcán suscita rechazo porque "arrastran algunas letras" o "acentúan mucho la ere" (Flores Mejía 2014: 437); "hablan cantadito" dicen, por ejemplo, informantes hondureños, respecto a la variedad mexicana (Hernández 2014: 753).

Es evidente que muchos enjuiciamientos traslucen prejuicios y estereotipos lingüísticos, ejemplos de discriminación lingüística que, a diferencia de otros tipos de segregación de tipo sexual o racial, se manifiestan muy abiertamente entre la población no lingüista (Milroy y Milroy 1985). Tómese como ejemplo el rechazo que en Venezuela suscita la variedad cubana por motivos socioeconómicos y culturales: "no saben nada", "son pobres" y la de la propia región venezolana de Zulia ("no me gustan los maracuchos") (cfr. Coello Millán 2014: 1487-1488).

En líneas generales, cabe destacar también que es común encontrar entre las opiniones de muchos informantes una actitud purista y recelosa ante la diversidad lingüística, una minusvaloración que se hace muy visible en el caso de lenguas indígenas que conviven con el español, de las variedades fronterizas y de las propias de fenómenos de hibridación lingüísti- 
ca. A este respecto, se observa, por ejemplo, que los informantes puertorriqueños manifiestan un notable rechazo ante la variedad dominicana, propia de un importante grupo migratorio en Puerto Rico (Mojica de León 2014: 1313). En este contexto, considérense también las críticas que se vierten por parte de muchos hablantes bolivianos capitalinos ante el habla de las zonas más rurales del país, en las que abunda el contacto entre el español y el quechua o el aymara (Aguilar 2014: 83), o la estigmatización de que es objeto por parte de los hablantes de Montevideo la variedad fronteriza del norte, zona que limita con Brasil. Allí se dice "mezclan portugués y español”, "tienen acento raro" (García de los Santos 2014: 1372).

Es bien sabido que uno de los rasgos que define a una comunidad de habla es también la valoración social (prestigio - manifiesto o encubierto - y estigma) ante diferentes formas lingüísticas y sus respectivos hablantes, actitudes lingüísticas, en definitiva, en cuyo surgimiento han contribuido poderosamente las llamadas variables de tipo individual (sexo, edad, etc.), pero también las variables del entorno (influencia de la familia, la presión grupal, etc.). Entre estas últimas hay que contar también con las políticas implementadas por los organismos de reglamentación lingüística, los medios de comunicación y las instituciones educativas, que, debido a su poder, alcance y visibilidad, son determinantes en la configuración de los modelos de prestigio, de las variedades que se constituirán en prototipos lingüísticos. En este sentido, merece la pena detenerse brevemente en el cambio actitudinal que parece advertirse, por ejemplo, en Paraguay. Aunque no pueda hablarse de abandono de la situación diglósica entre el español y el guaraní, las políticas lingüísticas en favor de este último han contribuido al aumento de lealtad lingüística de los hablantes guaraníes ante su lengua y al surgimiento de actitudes muy favorables del grueso de la población ante el bilingüismo generalizado (Chiquito y Saldívar 2014).

\section{ESTANDARIZACIÓN PLURICÉNTRICA Y ACTITUDES LINGÜÍSTICAS}

Indudablemente, el carácter positivo o negativo de las actitudes confiere bien prestigio bien estigmatización a los elementos propiamente lingüísticos y, por extensión, sociales, un aspecto crucial para la vitalidad y el estatus social de las variedades y de sus respectivos hablantes y para el consecuente surgimiento de sentimientos de sobreestimación e infraestimación lingüísticas entre la población. Tómese como ejemplo el fuerte estigma de la velarización de /r/ en Puerto Rico (Mojica de León 2014: 1285), el rechazo del intercambio entre /r/, /d/ y /1/ por /1/ o /i/ en la República Dominicana (Severino Cerda 2014: 1332) o el voseo en Uruguay (García de los Santos 2014: 1368). No hay que olvidar, sin embargo, que, si bien las actitudes ayudan a predecir comportamientos lingüísticos y a actuar de un determinado modo, ello no implica que la actitud manifestada encuentre necesariamente su correlato en la actuación lingüística (Cooper 1989: 162). Así sucede, de hecho, en Puerto Rico, República Dominicana o Uruguay ante las variantes aludidas. Una misma persona puede mostrar desprecio hacia estas variantes, sin que ello implique que las destierre de su discurso. No resulta, pues, extraño que la norma prescriptiva tenga mayor alcance en las actitudes que en los comportamientos lingüísticos.

Íntimamente relacionada con esta cuestión está la inclinación que los hablantes sienten por la escritura, hasta el punto de que la oralidad se juzga no como otra forma de expresión y organización de la actividad lingüística, sino como un trasunto imperfecto y degenerado de esta, que es considerada el único modelo de corrección lingüística. A este hecho ha 
contribuido, indudablemente el sesgo escriptista de la misma tradición lingüística occidental, que ha construido muchas teorías y modelos explicativos sobre parámetros exclusivos de la lengua escrita (Milroy 2001). Juicios de valor como "los que viven en la costa no pronuncian algunas letras," (Morett 2014: 847); "no quitan letras, por ejemplo, no dicen 'poqqué', 'veddá"” (Sobrino Triana y otros 2014: 323) o "hablar sin faltas" (Severino Cerda 2014: 1331; Yraola 2014: 595) evidencian, precisamente, el grafocentrismo del hablante común ante los hechos del lenguaje ( $c f r$. Moreno Cabrera 2011). Seguramente, este hecho se vea favorecido por los años de instrucción formal de muchos de los entrevistados, $\mathrm{y}$, por lo tanto, por un mayor contacto con la norma prescrita.

No obstante, no es superfluo recordar que también los resultados del proyecto LIAS confirman que el seguimiento de la norma prescrita y la inclusión en el repertorio lingüístico de variantes lingüísticas prestigiadas abiertamente genera, por lo general, admiración y valoraciones positivas en términos de poder social (inteligencia, erudición, educación, etc.) ( $c f r$. Paredes y Cestero 2018), pero puede suscitar rechazo en términos de solidaridad y prestigio encubierto (simpatía, cercanía, diversión, sentido del humor, etc.). De hecho, los comentarios de muchos informantes traslucen el recelo que sienten ante hablantes del exogrupo que "se hacen los finos" (García de los Santos 2014: 1374) o que "hablan con una papa en la boca" (Rojas 2014: 139); “usted ya ve cómo hablan los ricos; ellos son los más groseros, siendo que para nosotros son gente de más dinero, con más estudios” (Morett 2014: 872). Muestran, por tanto, desconfianza ante el empleo de una variedad que les parece excesivamente alejada del modelo lingüístico de su endogrupo, cuyo orgullo identitario se refleja en comentarios del tipo "hablan como nosotros" (Coello Millán 2014: 1451), "los escucho muy parecidos" (Hernández 2014: 739), al referirse a otras variedades del español que sienten próximas.

\subsection{Hablar 'correctamente'}

Es claro que la corrección lingüística es un hecho externo al sistema y que no son características lingüísticas intrínsecas las que determinan la corrección o incorrección de las variantes lingüísticas, sino que se debe recurrir a criterios como la autoridad, la tradición y el prestigio para justificar las soluciones normativas adoptadas. Sin embargo, no está de más insistir en el carácter fuertemente prescriptivo que los hablantes otorgan a las lenguas y en las ideologías que lleva aparejada la linguistic complaint tradition, "which requires that in language use, as in other matters, things should be done in the 'right' way" (Milroy y Milroy 1985: 1). En efecto, la existencia de normas explícitas e implícitas de buen uso condiciona las actuaciones lingüísticas de los hablantes y, en este sentido, atendiendo a los datos que arroja el Proyecto LIAS, merece la pena destacar que la cultura lingüística hispánica otorga una gran relevancia al conocimiento y el seguimiento de las normas lingüísticas prescritas. Así, en la mayor parte de países hispanos ante la pregunta "¿Qué importancia tiene para usted hablar 'correctamente'?/¿Qué tan importante es hablar 'correctamente'”, los valores más elevados de la escala ('importante' y 'muy importante') sobrepasan en todos los casos el $90 \%$ ( $c f r$. Apéndice). A este respecto, destaca particularmente Cuba, con un 92,3\% de informantes que lo juzgan 'muy importante'.

A este respecto, conviene recordar, en palabras de Speicher y Bielanski (2000: 156), que "the classification of a usage as standard or non-standard may be affected more by notions of correctness held by the well educated, that is Standard Ideology, rather than the amount 
of exposure or prevalence of certain usages". Por tanto, no extraña que las alusiones al necesario seguimiento de los dictados y recomendaciones normativas de las academias de la lengua española y, muy especialmente, a la RAE, sean muy frecuentes entre las opiniones vertidas en las diferentes ciudades hispánicas: "porque tal vez constantemente se basan en la gramática y en el buen uso de las palabras según el Diccionario de la Real Academia" (Bernal et alii 2014: 218); "conocer (aplicar, emplear, respetar...) las reglas (norma) según enseñan en la escuela (según la Academia, la RAE)" (Sobrino Triana et alii 2014: 338); "tienen a la RAE que difunde y codifica la lengua"; "ellos dictan las leyes de cómo hablar" (Rojas 2014: 148). Importante es, no obstante, resaltar que no se antepone la sujeción a la norma prescrita y el mantenimiento de la corrección lingüística a la comunicación efectiva. La transmisión de ideas claras y coherentes y, en definitiva, el entendimiento es, para la mayoría de informantes, el fin prioritario. Asimismo, cabe mencionar que, entre las razones aportadas sobre la importancia de hablar correctamente priman las de cariz comunicativo, si bien aparece también secundariamente la necesidad de 'hablar bien' para mantener el estatus social, para mostrar educación y cortesía con el interlocutor.

Por lo que se refiere a los criterios lingüísticos que llevan a la consideración de la corrección e incorrección, los encuestados hispanohablantes aluden, fundamentalmente, a los rasgos fonéticos y a la riqueza y variedad del léxico ${ }^{1}$, lo cual era esperable, dado que son los niveles lingüísticos más permeables a la conciencia lingüística: "diferenciar los hiatos y no pronunciarlos en un solo golpe de voz: tualla por toalla" (Flores Mejía 2014: 440); "evitar los vicios de dicción", "usar palabras cultas" (Hernández 2014: 746); "hacer uso de todas las palabras, de todas las formas de expresión que tiene, en nuestro caso, el español que es, creo, un idioma muy vasto" (Morett 2014: 868); "es emplear palabras preferiblemente cultas o las aceptadas por la Real Academia; es decir, emplear términos correctos, que estén en el diccionario" (Tinoco 2014: 1031). Se enfatizan también, pero de manera secundaria, variables discursivas como la adecuación, la claridad y la sencillez: "usar los códigos y registros correctos para cada sitio" (Flores Mejía 2014: 441); "hablar correctamente es saber comunicarse oralmente, es decir, saber expresarse para así lograr la capacidad de persuasión” (Zamora Úbeda 2014: 969).

Otra de las constantes advertidas en los estudios actitudinales, en general, y en el mundo hispánico, en particular, es la importancia del habla urbana y de la capital como foco de irradiación lingüística en la configuración de la norma culta (Lope Blanch 1986: 12-13; Silva-Corvalán 2001 [1989]: 35, etc.). Así lo recoge, por ejemplo, García de los Santos (2014: 1369) en referencia a Uruguay: "la zona este y Montevideo son las de mayor preferencia cuando se trata de determinar la 'mejor' habla en el país"; “en la capital se habla bien", testimonia Hernández (2014: 740) para referirse a la variante de Tegucigalpa; para los guatemaltecos capitalinos también su variante "es la mejor del país" (Acevedo y Quesada Pacheco 2014: 652). Sin embargo, también existen casos en los que surge una norma endógena que se alza en modelo de referencia frente a la variedad capitalina. Es, por ejemplo, lo que sucede en el área andina venezolana (Táchira), cuyos hablantes no consideran el habla caraqueña el modelo prestigioso (Coello Millán 2014: 1441). Pensemos también en

1 Nuestro propósito en este artículo es atender a las manifestaciones actitudinales generales de los diferentes países hispanos de los que se elicitaron datos lingüísticos en el marco del Proyecto LIAS. No nos detendremos en esta ocasión en analizar los resultados según las diferentes variables de estratificación (edad, sexo, nivel de instrucción). 
el particular caso de España, donde la mayor ejemplaridad lingüística se sigue atribuyendo al modelo centro-septentrional castellano ( $c f r$. Fernández Juncal y Amorós-Negre 2014; Paredes y Cestero 2018). En todo caso, este hecho no obsta para que este estándar nacional conviva con otras variedades prestigiosas a distintos niveles, como ocurre con la emergencia de estándares regionales o dialectos terciarios, según ha explicado Villena Ponsoda (2008) a propósito de las variedades ejemplares de la Andalucía oriental, las cuales convergen en ciertos rasgos con el modelo centro-septentrional del español europeo a la vez que mantienen otros rasgos típicamente meridionales (cfr. Hernández Campoy y Villena Ponsoda 2009; Villena Ponsoda y Ávila Muñoz 2014; Villena Ponsoda y Vida Castro 2017; Méndez García de Paredes y Amorós-Negre 2018).

\subsection{La concepción normativa y la unidad en la diversidad}

Otra cuestión fundamental atiende, precisamente, a la concepción normativa del español que tiene el grueso de hispanohablantes encuestados. En efecto, nos interesa indagar en la representación actitudinal que manifiestan hacia la norma del español y la configuración de su propia identidad lingüístico-cultural. En este sentido, resulta crucial analizar si, a la luz de los datos del Proyecto LIAS, puede afirmarse que el pluricentrismo que se reconoce en los círculos académicos se refleja en las creencias de la mayoría de la población nativa de las capitales de la hispanofonía. Para ello, conviene detenerse en las respuestas dadas en las distintas naciones a las preguntas: "Diga/Mencione un país en que se hable español/castellano, en donde, para usted (o desde su punto de vista) se hable más 'correctamente' e 'incorrectamente' ".

A este respecto, téngase en cuenta que hablar de pluricentrismo implica, tal y como se anticipó anteriormente, hacerlo en términos de ejemplaridad comunicativa. El español es considerado una lengua pluricéntrica (Thompson 1992; Rivarola 2001; Oesterreicher 2001, etc.) y lo es, sin duda, porque en ella pueden reconocerse sin dificultad varios núcleos que proyectan sus modos propios de uso de la lengua, diferentes normas cultas en sus respectivos dominios idiomáticos. Así, teniendo en cuenta los datos del Proyecto, el pluricentrismo lingüístico encuentra todavía poco reflejo en el plano actitudinal de la mayoría de la población nativa hispanohablante. La idea de que el mejor español es el de "la madre patria", "la cuna del idioma" está presente en mayor o menor medida en la mayoría de los análisis de las percepciones lingüísticas de los hablantes capitalinos encuestados, si bien es cierto que hay países que le otorgan cada vez un carácter más modélico a sus variedades nacionales, que son apreciadas no solo en términos de solidaridad sino también de poder.

Tal y como puede observarse en el apéndice, España continúa siendo el país que recibe un mayor número de alusiones en cuanto a corrección lingüística. Todos los países la incluyen entre la nómina de naciones en las que se habla 'más correctamente' el español y en 15 de ellos -España incluido- ocupa la primera posición. En países como Costa Rica, Cuba, El Salvador, Honduras y Nicaragua, los porcentajes que señalan a España como el modelo lingüístico en más alto grado superan el 50\%. Únicamente Colombia, México y Venezuela se autocategorizan como naciones en las que se habla 'más correctamente' el español, aunque secundariamente aludan también al modelo peninsular. Se observa, pues, una mayor seguridad lingüística y el orgullo nacional de estos hablantes ante su propia variedad lingüística, a la que le confieren prestigio y legitimidad como medio más apropiado también para los ámbitos propios del estándar, esto es, las situaciones formales de la distancia comunicativa. 
Tras España, merece la pena aludir al caso de Colombia, país que despunta - es mencionado 12 veces por el resto de naciones - como prototipo de corrección lingüística. Se observa, por tanto, que continúan vigentes los mitos tan extendidos de España y Colombia como depositarias del 'hablar bien' (Moreno Fernández 2005: 211), donde, a juicio de los informantes, hay un mayor seguimiento de la norma y se garantiza una mayor comprensión lingüística ${ }^{2}$ con esa supuesta 'neutralidad' de la pronunciación: "sé que el habla ahí es un poco mejor. "Me lo han dicho en la escuela", comentan, por ejemplo, informantes mexicanos (Morett 2014: 885), una opinión que trasluce el prescriptivismo y el eurocentrismo que ha caracterizado largo tiempo la enseñanza tradicional de la lengua en Hispanoamérica ( $c f r$. Arnoux 1999; Blanco de Margo 1999).

Inversamente, cabe poner de relieve que son México — mencionado por 9 países-, seguido de Argentina - 8 menciones_- los considerados por el resto de naciones hispanohablantes 'países en los que se habla más incorrectamente'. Debe tomarse en consideración, no obstante, que los países más referenciados para bien o para mal son también los más conocidos, los que se tienen más presentes por circunstancias históricas, socioculturales o económicas. En efecto, a propósito del elevado porcentaje que obtiene México en los concerniente a la incorrección lingüística entre los encuestados salvadoreños, Rivera Orellana (2014: 517-518) comenta lo siguiente:

Se deben tomar en cuenta una serie de factores de carácter histórico cuando en El Salvador se habla de México. Primero, que hay factores sociales que predisponen a la opinión pública, y uno de los más importantes es el tema de la emigración. El último informe del Pew Hispanic Center (Álvarez, 30 de junio, 2012) señala que hay 1,8 millones de salvadoreños que viven en Estados Unidos. Son así una de las cinco minorías más importantes de latinos en ese país de Norteamérica, y México es un espacio por el que transitan no solo los salvadoreños, sino los centroamericanos que ilegalmente llegan a Estados Unidos [..]. Otro factor para tomar en cuenta es la fuerte presencia de programas de la televisión mexicana en los canales locales. Por ello, es fácil entender que los salvadoreños están muy familiarizados con el modo de hablar de los mexicanos $[\ldots]$

En este mismo sentido, la mayor o menor relación entre las diferentes naciones (vecindad, rivalidad, prestigio histórico, etc.) explica, asimismo, la dualidad y polarización de valores en los datos obtenidos respecto a la corrección lingüística. Un mismo país es en ocasiones uno de los más referenciados tanto en la pregunta sobre localización de la corrección lingüística como de la incorrección lingüística. Así ocurre, en efecto, en Colombia, Cuba, Ecuador, El Salvador y España (cfr. Apéndice).

En todo caso, debe enfatizarse que ha habido un aumento de la conciencia sobre la diversidad lingüística y, lo que es más importante, sobre la riqueza que esto supone. El hecho de que haya hablantes que apuntan a que ningún país habla más o menos (in)correctamente que otro, sin duda, es un indicio de una mayor equipolencia y simetría entre las diferentes normas nacionales de la hispanofonía, al menos, actitudinalmente. Particularmente intere-

2 Otra cuestión interesante atiende a la correlación existente entre los factores lingüísticos y extralingüísticos en la valoración sobre la corrección o incorrección idiomáticas en las diferentes naciones hispánicas, así como la propia relación entre las percepciones cognitivas y afectivas de los hablantes capitalinos de las diversas urbes hispánicas, como ha mostrado Rojas (2012) para Chile. 
sante es el dato aportado por Argentina ${ }^{3}$ : un 34\% de los informantes seleccionaron la opción 'ninguno' en respuesta a la pregunta de qué países hablan más correctamente y un $25 \%$ en referencia a las naciones que hablan 'peor', una opción más acorde con la opción pluricéntrica que, en palabras de Llull y Pinardi (2014: 36), crece entre los segmentos de menor edad y de mayor nivel educativo.

Otro dato interesante e íntimamente vinculado a la corrección y a la localización de los modelos idiomáticos en el mundo hispanohablante es el debate sobre la unidad de la lengua. Lejos del miedo por una fragmentación interna del sistema lingüístico español, el recurso a la unidad en la diversidad es, en la actualidad, un leitmotiv de los discursos que exaltan el español como lengua que en su devenir histórico ha permitido la expresión de las singularidades de las diversas naciones, al tiempo que ha posibilitado el entendimiento y concordia entre todas ellas ( $c f r$. Del Valle 2007). Así se refleja, de hecho, en el renovado lema de las academias de la lengua española unifica, limpia y fija, acorde con la política lingüística panhispánica. No obstante, cabe preguntarse por las creencias de los hispanohablantes al respecto y hasta qué punto la idea de unidad de la mancomunidad panhispánica lleva aparejada la homogeneización lingüística. Así, a la pregunta “¿Sería bueno que todos habláramos el mismo español/castellano (en los países donde se habla)?”, 7 países rechazan tal propuesta en más de un 50\% ( $c f r$. Apéndice). Destaca, sin duda, la oposición que origina la idea en países como Argentina y México, donde un $60 \%$ de los informantes se manifiesta en contra de la uniformidad lingüística porque se perdería la riqueza idiomática, la idiosincrasia dialectal y la propia identidad etnolingüística: "sería aburrido"; "la cultura es hermosa en su diversidad"; "tenemos que tener algo que nos diferencie", comentan al respecto algunos encuestados panameños (Tinoco 2014: 1037).

Contrariamente, muy favorables a la homogeneización lingüística son, a la luz de los datos analizados, República Dominicana, Ecuador, Costa Rica y Perú, cuyos informantes resaltaron el valor puramente pragmático y utilitario de la lengua, de forma que cuanto más homogéneo fuera mayor sería el entendimiento y la comunicación: "no entendemos las palabras que ellos usan, ni ellos nos entienden nuestras palabras", argumentan algunos informantes ecuatorianos sobre sus experiencias en España (Flores Mejía 2014: 452). Para otros ciudadanos peruanos, la uniformidad lingüística sería positiva porque "predominaría el uso del español y se relegaría el uso de otras lenguas a contextos específicos familiares o técnicos"; "tendríamos el mismo castellano, que conlleva a tener el mismo dejo, eso evitaría que se crearan barreras sociales al tratar a personas de otros pueblos o países" (Arias 2014: 1223). Parece, además, advertirse que las personas de mayor edad y menor nivel sociocultural de República Dominicana, Ecuador, Costa Rica y Perú son las más proclives a valorar positivamente la mencionada homogeneización (Severino Cerda 2014: 1335-1336; Flores Mejía 2014: 450-451; Calvo Shadid y Castillo Rivas 2014: 269; Arias 2014: 1221-1222).

\section{CONCLUSIONES}

En una época en la que la lingüística hispánica ha tomado conciencia del carácter pluricéntrico de la lengua española, esto es, de la existencia de diversos modelos idiomáticos

3 Porcentajes también reseñables de la opción 'ninguno, en alusión a los países que hablan el español 'más o menos correcto', son los aportados por Bolivia, El Salvador, México o Uruguay ( $c f r$. Apéndice). 
y normas cultas de referencia en el mundo hispánico, y se debate en torno al carácter más o menos dominante de unas variedades frente a otras, los estudios actitudinales deben considerarse fundamentales. Este trabajo constituye un ejemplo del valor de las creencias y actitudes lingüísticas como indicadores explicativos de muy diversos comportamientos sociales y lingüísticos. Se ha puesto de relieve que la medición de actitudes puede ayudar a caracterizar la percepción del grado de estandarización de determinadas variantes y variedades de las lenguas y contribuye a explicar muchos comportamientos de hipercorrección e inseguridad lingüística de los hablantes, siempre preocupados por el mantenimiento de la higiene verbal (Cameron 1995).

En las páginas precedentes se ha enfatizado que la reivindicación del pluralismo y la diversidad lingüística no puede hacer olvidar a la ciencia lingüística que, por razones extralingüísticas, tal equipolencia no suele reflejarse en la vida social de las lenguas y, por consiguiente, las actitudes lingüísticas de la población, en general, y de los hispanohablantes, en particular, muestran que son unas pocas variedades las que gozan de mayor reconocimiento y prestigio social, económico y/o cultural. Los hablantes poseen una inclinación prescriptiva, de forma tal que tienden a valorar los hechos del lenguaje como 'buenos y malos', 'mejores y peores', y a pensar que la lengua espontánea es una degeneración de una lengua culta elaborada, mitos lingüísticos que los profesionales de la lingüística y de la educación debemos tratar de desterrar.

Así pues, pese a la persistencia en las creencias de los hispanohablantes de una notable asimetría a favor del español peninsular frente al resto de variedades nacionales del español americano, el análisis de las creencias de los hispanohablantes capitalinos en torno a la corrección y la unidad lingüísticas en el mundo hispánico arroja resultados muy interesantes y esclarecedores en términos de pluricentrismo lingüístico. Las tendencias actitudinales advertidas muestran que los hispanohablantes están legitimando cada vez más sus variedades nacionales autóctonas y reivindicando su endonormatividad — particularmente reseñables son los resultados de las encuestas en Colombia, México, Venezuela y Argentina- Por ello $\mathrm{y}$, a fin de que las diversas variedades hispánicas logren una estabilización normativa plena y una verdadera equipolencia, deben realizarse más esfuerzos por parte de los diferentes agentes de reglamentación lingüística en los distintos países hispánicos, cuyas acciones son determinantes en la configuración de las ideologías lingüísticas.

\section{REFERENCIAS BIBLIOGRÁFICAS}

Albarracín, D., B. T. Thompson y M. P. Zanna (2005). The Handbook of Attitudes. New York/London: Laurence Erlbaum.

Acevedo, A. L. y M. Á. Quesada Pacheco (2014). “Actitudes lingüísticas en Guatemala. Creencias y actitudes lingüísticas respecto al español de los chapines capitalinos”. En Chiquito, A. B. y M. Á. Quesada Pacheco (eds.). Actitudes lingüísticas de los hispanohablantes hacia el idioma español y sus variantes. Bergen: Bergen Language and Linguistic Studies (BeLLS), 5, pp. 637-714.

Aguilar, M. J. (2014). "Actitudes lingüísticas hacia el castellano en Bolivia. Entre la fidelidad y la conciencia lingüística”. En Chiquito, A. B. y M. Á. Quesada Pacheco (eds.). Actitudes lingüísticas de los hispanohablantes hacia el idioma español y sus variantes. Bergen: Bergen Language and Linguistic Studies (BeLLS), 5, pp. 63-121.

Alvar, M. (dir.) (1996a). Manual de dialectología hispánica. El español de España. Barcelona: Ariel. Alvar, M. (dir.) (1996b). Manual de dialectología hispánica. El español de América. Barcelona: Ariel 
Amorós-Negre, C. y E. Prieto de los Mozos (2017). "El grado de pluricentrismo de la lengua española”, Language Problems and Language Planning, 41 (3), pp. 245-264. DOI: 10.1075/lplp.00004. amo.

Arias, A. G. (2014). “Actitudes lingüísticas en el Perú. Predominancia del castellano de las costa central y norte”. En Chiquito, A. B. y M. Á. Quesada Pacheco (eds.). Actitudes lingüísticas de los hispanohablantes hacia el idioma español y sus variantes. Bergen: Bergen Language and Linguistic Studies (BeLLS), 5, pp. 1185-1248.

Arnoux, E. N. (1999). "El discurso normativo en los textos gramaticales de Andrés Bello". En Arnoux, E. N. y R. Bein (comps.). Prácticas y representaciones del lenguaje. Buenos Aires: Eudeba, pp. 38-61.

Arnoux, E. N. y J. Del Valle (2010). "Las representaciones ideológicas del lenguaje: Discurso glotopolítico y panhispanismo", Spanish in context, 7 (1), pp. 1-24.

Auer, P., F. Hinskens y P. Kerswill (eds.) (2005). Dialect Change: Convergence and Divergence in European Languages. Cambridge: Cambridge University Press.

Bernal, J., A. Munévar y C. Barajas (2014). "Actitudes lingüísticas en Colombia”. En Chiquito, A. B. y M. Á. Quesada Pacheco (eds.). Actitudes lingüísticas de los hispanohablantes hacia el idioma español y sus variantes. Bergen: Bergen Language and Linguistic Studies (BeLLS), 5, pp. 189-245.

Blanco de Margo, M. (1999). "La configuración de la lengua nacional en los orígenes de la escuela secundaria argentina”. En Arnoux, E. N. y R. Bein (comps.). Prácticas y representaciones del lenguaje. Buenos Aires: Eudeba, pp. 75-100.

Blas Arroyo, J. L. (1999). "Las actitudes hacia la variación intradialectal en la sociolingüística hispánica", Estudios filológicos, 34, pp. 47-72.

Bugel, T. (2014) (ed.). "Nuevos estudios sobre actitudes lingüísticas", Revista Internacional de Lingüística Iberoamericana, 23 (1).

Calvo Shadid, A. y J. Castillo Rivas (2014). "Las actitudes lingüísticas en el español de San José, Costa Rica”. En Chiquito, A. B. y M. Á. Quesada Pacheco (eds.). Actitudes lingüisticas de los hispanohablantes hacia el idioma español y sus variantes. Bergen: Bergen Language and Linguistic Studies (BeLLS), 5, pp. 246-289.

Cameron, D. (1995). Verbal Hygiene. London/New York: Routledge.

Caravedo, R. (2014). Percepción y variación lingüística. Madrid/Frankfurt am Main: Iberoamericana/ Vervuert.

Cargile, A, H. Giles, E. B. Ryan y J. J. Bradac (1994). "Language attitudes as a social process: A conceptual model and new directions", Language \& Communication, 14 (3), pp. 211-236.

Cestero, A. M. y Fl. Paredes (2013). "Metodología PRECAVESXXI. Proyecto para el estudio de creencias y actitudes hacia las variedades del español en el siglo XXI": http://www.variedadesdelespanol.es/Content/Metodolog\%C3\%ADa\% 20proyect o\%20PRECAVES-XXI.pdf. (05-012019).

Cestero, A. M. y Fl. Paredes (2015a). "Creencias y actitudes hacia las variedades normativas del español actual. Primeros resultados del Proyecto PRECAVES-XXI", Spanish in Context, 12 (2), pp. 255-279.

Cestero Mancera, A.M. y Fl. Paredes García (2015b). "Creencias y actitudes hacia las variedades del español en el siglo XXI: avance de un proyecto de investigación”. En Da Hora, D., J. Lopes, R. Pedrosa y R. de Lucena (eds.). ALFAL 50 anos: contribuições para os estudos linguísticos e filológicos. João Pessoa: Ideia, pp. 652-683.

Chiquito, A. B. y M. C. Saldívar Dick (2014). "Actitudes lingüísticas en Paraguay. Identidad lingüística de los hablantes de lengua materna castellana en Asunción”. En Chiquito, A. B. y M. Á. Quesada Pacheco (eds.). Actitudes lingüísticas de los hispanohablantes hacia el idioma español y sus variantes. Bergen: Bergen Language and Linguistic Studies (BeLLS), 5, pp. 1065-1184.

Chiquito A. B. y M. Á. Quesada Pacheco ed. (2014). Actitudes lingüisticas de los hispanohablantes hacia el idioma español y sus variantes. Bergen: Bergen Language and Linguistic Studies (BeLLS), 5. 
Coello M. y H. Yosibel (2014). “Actitudes lingüísticas en Venezuela. Exploración de creencias hacia la variante nacional, la lengua española y el español dialectal”. En Chiquito, A. B. y M. Á. Quesada Pacheco (eds.). Actitudes lingüisticas de los hispanohablantes hacia el idioma español y sus variantes. Bergen: Bergen Language and Linguistic Studies (BeLLS), 5, pp. 1407-1532.

Cooper, R. L. (1989). Language Planning and Social Change. Cambridge: Cambridge University Press.

Del Valle, José (ed.) (2007). La lengua, ¿patria común?. Ideas e ideologías del español. Madrid/ Frankfurt am Main: Iberoamericana/Vervuert.

Fernández-Juncal, C. y C. Amorós-Negre (2014). "Polarización y tensión normativas: Actitudes hacia la norma prescrita en el español peninsular centro-septentrional". En Zimmermann, Kl (ed.). Prácticas y políticas lingüisticas. Nuevas variedades, normas, actitudes y perspectivas. Madrid: Iberoamericana Vervuert, pp. 231-256.

Flores Mejía, E. (2014). “Actitudes lingüísticas en Ecuador. Una tradición normativa que subsiste”. En Chiquito, A. B. y M. Á. Quesada Pacheco (eds.). Actitudes lingüísticas de los hispanohablantes hacia el idioma español y sus variantes. Bergen: Bergen Language and Linguistic Studies (BeLLS), 5, pp. 409-488.

García de los Santos, E. (2014). "Actitudes lingüísticas en Uruguay. Tensiones entre la variedad y la identidad”. En Chiquito, A. B. y M. Á. Quesada Pacheco (eds.). Actitudes lingüísticas de los hispanohablantes hacia el idioma español y sus variantes. Bergen: Bergen Language and Linguistic Studies (BeLLS), 5, pp. 1346-1406.

Gimeno, F. (1993 [1990]). Dialectología y sociolingüistica españolas. Alicante: Universidad de Alicante.

Hernández Campoy, J.M. y J. A. Villena Ponsoda (2009). "Standardness and Non-Standardness in Spain: Dialect Attrition and Revitalization of Regional Dialects of Spanish", International Journal of the Sociology of Language, 196/197, pp. 181-214.

Hernández, H. (2014). "Actitudes lingüísticas en Honduras. Un estudio sociolingüístico sobre el español de Honduras frente al de otros países de habla hispana”. En Chiquito, A. B. y M. Á. Quesada Pacheco (eds.). Actitudes lingüísticas de los hispanohablantes hacia el idioma español y sus variantes. Bergen: Bergen Language and Linguistic Studies (BeLLS), 5, pp. 715-792.

Lara, L. F. (2004). Lengua histórica y normatividad. México: El Colegio de México.

Lope Blanch, J. M. (1986). El estudio del español hablado culto. Historia de un proyecto. México: UNAM.

López Morales, H. (2004 [1989]). Sociolingüistica. Madrid: Gredos.

Llull, G. y L. C. Pinardi (2014). "Actitudes lingüísticas en la Argentina. El español en Buenos Aires: Una aproximación a las representaciones de sus hablantes". En Chiquito, A. B. y M. Á. Quesada Pacheco (eds.). Actitudes lingüisticas de los hispanohablantes hacia el idioma español y sus variantes. Bergen: Bergen Language and Linguistic Studies (BeLLS), 5, pp. 1-62.

Méndez-Ga de Paredes, E. y C. Amorós-Negre (2018). "The status of Andalusian in the Spanish-speaking world: is it currently possible for Andalusia to have its own linguistic standardization process?", Current Issues in Language Planning 20, 179-198. DOI: 10.1080/14664208.2018.1495369.

Milroy, J. y L. Milroy (1985). Authority in language. Investigating language prescription \& standardization. London: Routledge.

Milroy, J. (2001). "Language ideologies and the consequences of standardization", Journal of Sociolinguistics, 5 (4), pp. 530-555.

Mojica De León, C. M. (2014). "Una mirada hacia las actitudes lingüísticas en Puerto Rico”. En Chiquito, A. B. y M. Á. Quesada Pacheco (eds.). Actitudes lingüisticas de los hispanohablantes hacia el idioma español y sus variantes. Bergen: Bergen Language and Linguistic Studies (BeLLS), 5, pp. 1249-1315.

Moreno Cabrera, J. C. (2011). “ 'Unifica, limpia y fija'. La RAE y los mitos del nacionalismo lingüístico español". En Senz, S. y M. Alberte (eds.). El dardo en la Academia. Esencia y vigencia de las academias de la lengua española. Vol. I. Barcelona: Melusina, pp. 156-341. 
Moreno Fernández, F. (2005). Historia social de las lenguas de España. Barcelona: Ariel.

Moreno Fernández, F. (2012). Sociolingüistica cognitiva. Proposiciones, escolios y debates. Madrid/ Frankfurt am Main: Iberoamericana/Vervuert.

Morett, S. (2014). “Actitudes lingüísticas en México. Entre el chovinismo y el malinchismo”. En Chiquito, A. B. y M. Á. Quesada Pacheco (eds.). Actitudes lingüisticas de los hispanohablantes hacia el idioma español y sus variantes. Bergen: Bergen Language and Linguistic Studies (BeLLS), 5, pp. 793-933.

Muhr, R. (2012). "Linguistic dominance and non-dominance in pluricentric languages. A typology". En Muhr, R. (ed.). Non-dominant Varieties of Pluricentric Languages. Getting the Picture. In memory of professor Michael Clyne. Wien: Peter Lang Verlag, pp. 23-48.

Niedzielsky, N. y D. Preston (2000). Folk linguistics. New York: Mouton.

Oesterreicher, W. (2001). "Plurizentrische Sprachkultur der Varietätenraum des Spanischen”, Romanistisches Jahrbuch, 51, pp. 281-311.

Paredes García, F. y A.M. Cestero Mancera (2018). "Percepción de las variedades cultas del español por hablantes del centro-norte de España según los datos del proyecto PRECAVES XXI: el español ejemplar y la variedad propia”, Oralia, 21 (1), pp. 87-112.

Preston, D. (1989). Perceptual Dialectology. Non linguists'view of Areal Linguistics. Dordrecht: De Gruyter.

Rivarola, J. L. (2001). "Las normas regionales y socioculturales. La variación lingüística. Sobre variedades y normas del español en el marco de una cultura lingüística pluricéntrica", II Congreso Internacional de la Lengua Española: http://congresosdelalengua.es/valladolid/ponencias/ unidad_diversidad_del_espanol/1_la_norma_hispanica/rivarola_j.htm (13-01-2019).

Rivera Orellana, E. (2014). "Actitudes lingüísticas de los hablantes de San Salvador, El Salvador". En Chiquito, A. B. y M. Á. Quesada Pacheco (eds.). Actitudes lingüísticas de los hispanohablantes hacia el idioma español y sus variantes. Bergen: Bergen Language and Linguistic Studies (BeLLS), 5, pp. 489-550.

Rojas, D. (2012). "Actitudes lingüísticas de hispanohablantes de Santiago de Chile: creencias sobre la corrección idiomática", Onomázein, 26 (2), pp. 69-98: http://onomazein.letras.uc.cl/Articulos/26/3_Rojas.pdf (13-01-2019).

Rojas, D. (2014). "Actitudes lingüísticas en Santiago de Chile”. En Chiquito, A. B. y M. Á. Quesada Pacheco (eds.). Actitudes lingüisticas de los hispanohablantes hacia el idioma español y sus variantes. Bergen: Bergen Language and Linguistic Studies (BeLLS), 5, pp. 122-188.

Ryan, E. B. y H. Giles (eds.) (1982). Attitudes towards language variation. Social and applied contexts. London: Edward Arnold.

Severino Cerda, Gl. M. (2014). "Actitudes lingüísticas en República Dominicana. Conciencia e identidad lingüística en la ciudad de Santo Domingo". En Chiquito, A. B. y M. Á. Quesada Pacheco (eds.). Actitudes lingüísticas de los hispanohablantes hacia el idioma español y sus variantes. Bergen: Bergen Language and Linguistic Studies (BeLLS), 5, pp. 1316-1345.

Silva-Corvalán, C. (2001 [1989]). Sociolingüística. Teoría y análisis. Madrid: Alhambra.

Sobrino Triana, R., L. E. Montero Bernal y A. J. Menéndez Pryce (2014). "Actitudes lingüísticas en Cuba. Cambios positivos hacia la variante nacional de lengua”. En Chiquito, A. B. y M. Á. Quesada Pacheco (eds.). Actitudes lingüisticas de los hispanohablantes hacia el idioma español y sus variantes. Bergen: Bergen Language and Linguistic Studies (BeLLS), 5, pp. 1290-1408.

Speicher, B. L. y J. R. Bielanski (2000). "Critical Thoughts on Teaching Standard English", Curriculum Inquiry, 30 (2), pp. 147-169.

Stewart, W. (1968). "A sociolinguistic typology for describing national multilingualism". En Fishman, J.A. (ed.). Readings in the Sociology of Language. The Hague: Mouton, pp. 531- 545.

Thompson, R.W. (1992). "Spanish as a pluricentric language". En Clyne, M. (ed.). Pluricentric Languages: differing norms in different nations. Berlin/New York: Mouton de Gruyter, pp. 45-70. 
Tinoco, T. (2014). “Actitudes lingüísticas en Panamá. Incursión en la percepción sociolingüística y la valoración de la lengua por los hispanohablantes panameños". En Chiquito, A. B. y M. Á. Quesada Pacheco (eds.). Actitudes lingüísticas de los hispanohablantes hacia el idioma español y sus variantes. Bergen: Bergen Language and Linguistic Studies (BeLLS), 5, pp. 1011-1064.

Villena Ponsoda, J. A. (2008). "Divergencia dialectal en el español de Andalucía: el estándar regional y la nueva koiné meridional”. En Döhla, H.J., R. Montero Muñoz y F. Báez de Aguilar (eds.). Lenguas en diálogo. El iberorromance y su diversidad lingüística y literaria. Ensayos en homenaje a Georg Bossong, Madrid/Frankfurt am Main: Iberoamericana/Vervuert, pp. 369-391

Villena Ponsoda, J. A. y A. Ávila Muñoz (2014). "Dialect stability and divergence in southern Spain. Social and personal motivations". En Braunmüller, K., S. Höder y K. Kühl (eds.). Stability and divergence in language contact. Factors and mechanisms. Amsterdam: John Benjamins, SILV 16, pp. 207-238.

Villena Ponsoda, J. A. y M. Vida Castro (2017). "Between local and standard varieties: horizontal and vertical convergence and divergence of dialects in Southern Spain”. En Buchstaller, I. y B. Siebenhaar (eds.). Language Variation. European Perspectives. Selected papers from the Eighth International Conference on Language Variation in Europe (ICLaVE 8). Amsterdam: John Benjamins, pp. 125-140. DOI: 10.1075/silv.19.08vil.

Volóshinov, V. (1986). Marxism and the philosophy of language. Cambridge: Harvard University Press.

Woolard, K. A. (2008). "Les ideologies lingüístiques: una visió general d'un camp des de l'antropologia lingüística", Revista de Llengua i Dret, 49, pp. 179-199.

Yraola, A. (2014). "Actitudes lingüísticas en España”. En Chiquito, A. B. y M. Á. Quesada Pacheco (eds.). Actitudes lingüisticas de los hispanohablantes hacia el idioma español y sus variantes. Bergen: Bergen Language and Linguistic Studies (BeLLS), 5, pp. 551-636.

Zamora Úbeda, Z. C. (2014). "Actitudes lingüísticas de los hablantes de Managua, Nicaragua”. En Chiquito, A. B. y M. Á. Quesada Pacheco (eds.). Actitudes lingüísticas de los hispanohablantes hacia el idioma español y sus variantes. Bergen: Bergen Language and Linguistic Studies (BeLLS), 5, pp. 934-1010 (02-02-2019). 
24 Percepción lingüística y pluricentrismo: AnÁlisis del binomio a la luZ de los Resultados del Proyecto....

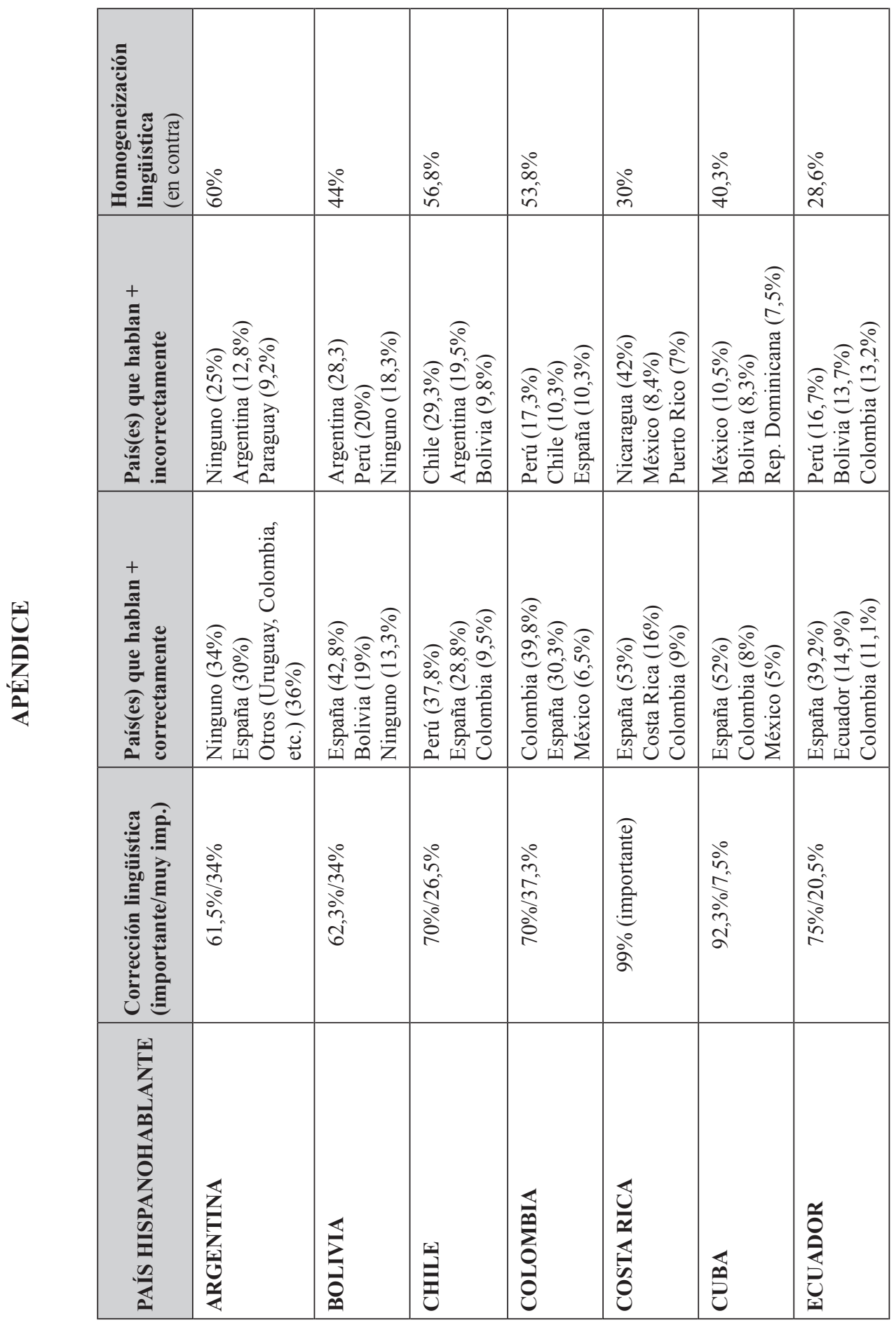




\begin{tabular}{|c|c|c|c|c|c|c|c|c|}
\hline 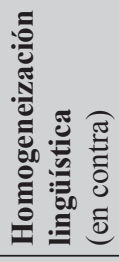 & $\stackrel{\circ}{\circ}$ & $\begin{array}{l}\stackrel{0}{~} \\
\text { 字 }\end{array}$ & $\frac{\partial^{\circ}}{b^{\circ}}$ & $\begin{array}{l}\text { के } \\
\text { ले }\end{array}$ & ठ̊ㅇㅇ & $\stackrel{\circ}{P^{\circ}}$ & $\begin{array}{l}\partial^{\circ} \\
\infty \\
0 \\
0\end{array}$ & $\begin{array}{l}\stackrel{0}{0} \\
\text { ñ }\end{array}$ \\
\hline 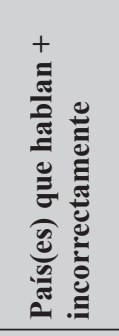 & 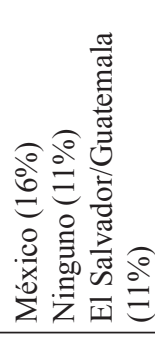 & 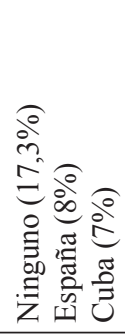 & 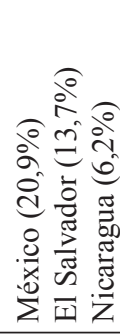 & 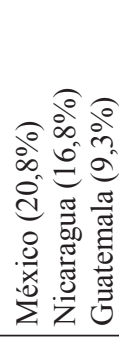 & 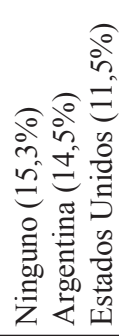 & 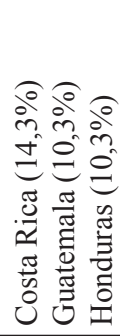 & 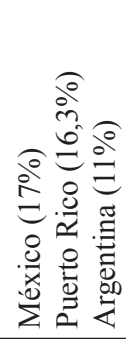 & 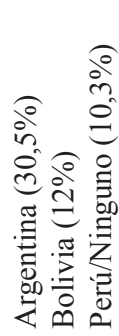 \\
\hline 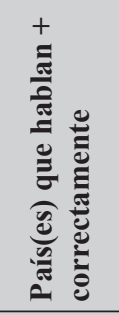 & 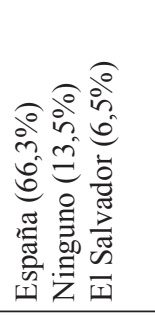 & 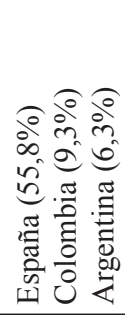 & 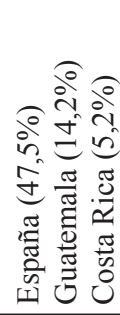 & 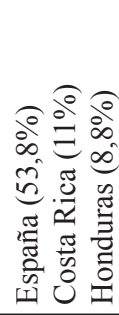 & 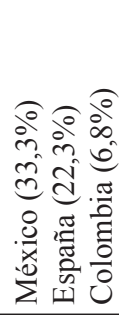 & 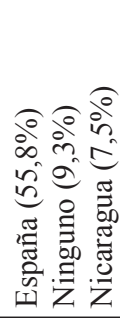 & 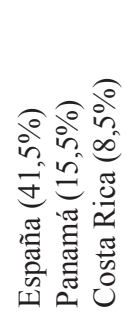 & 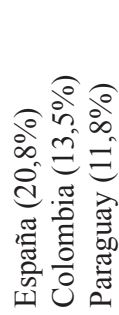 \\
\hline 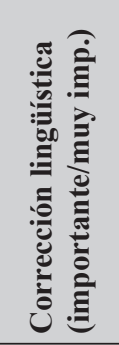 & $\underset{\stackrel{\circ}{\circ}}{\stackrel{\circ}{\circ}}$ & 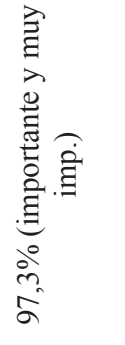 & $\begin{array}{l}\partial_{0}^{\circ} \\
\text { हे } \\
\frac{\partial}{0} \\
\overrightarrow{0}\end{array}$ & 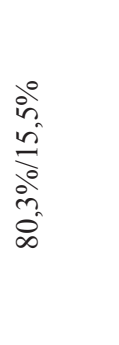 & $\begin{array}{l}\text { oे } \\
\text { mo } \\
\text { in } \\
\text { î }\end{array}$ & $\begin{array}{l}\frac{\infty}{\infty} \\
\vec{i} \\
0 \\
i n \\
i \\
i\end{array}$ & $\begin{array}{l}\stackrel{0}{\circ} \\
\frac{1}{0} \\
\infty \\
\infty \\
\infty\end{array}$ & $\begin{array}{l}\frac{0}{0} \\
\frac{0}{0} \\
\stackrel{0}{\infty} \\
\stackrel{0}{2}\end{array}$ \\
\hline 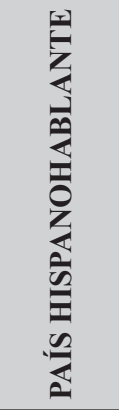 & 产 & 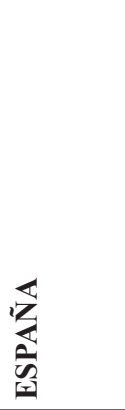 & 岁 & 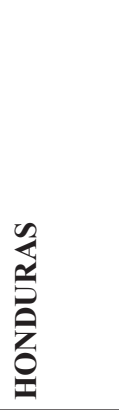 & 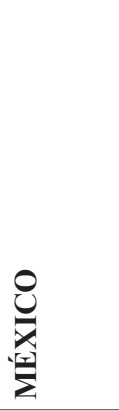 & 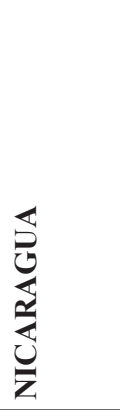 & 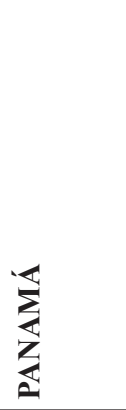 & 悹 \\
\hline
\end{tabular}


Percepción lingüística y pluricentrismo: AnÁlisis del binomio a la luZ de los Resultados del Proyecto...

\begin{tabular}{|c|c|c|c|c|c|}
\hline 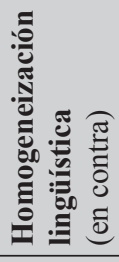 & 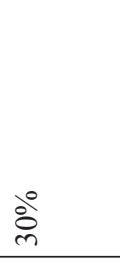 & $\begin{array}{l}\text { के } \\
\text { iे } \\
\dot{+}\end{array}$ & $\begin{array}{l}\text { bे } \\
\text { iे } \\
\text { i }\end{array}$ & ڤे & $\begin{array}{l}\stackrel{0}{ } \\
\text { テे } \\
\text { f }\end{array}$ \\
\hline 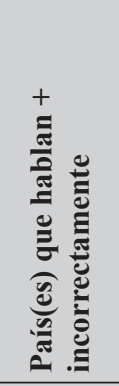 & 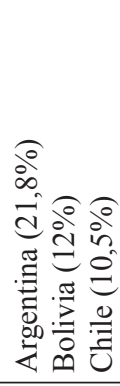 & 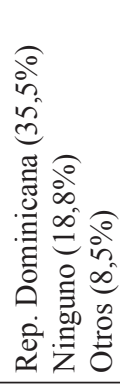 & 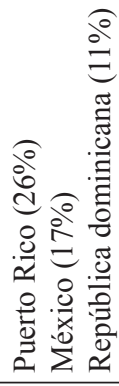 & 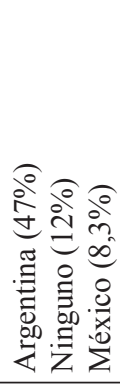 & 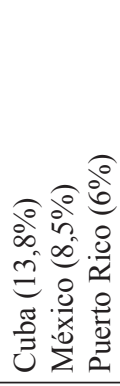 \\
\hline 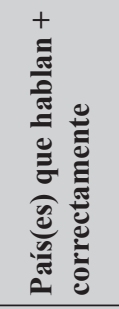 & 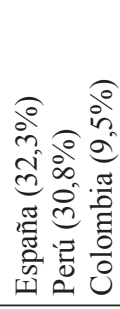 & 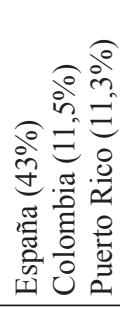 & 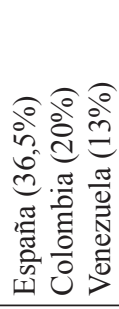 & 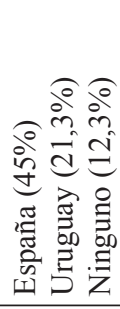 & 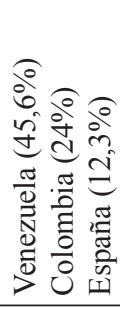 \\
\hline 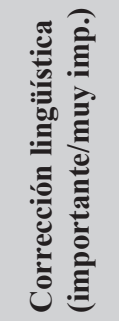 & $\begin{array}{l}\frac{0}{i n} \\
\frac{n}{0} \\
\infty \\
\infty \\
i\end{array}$ & 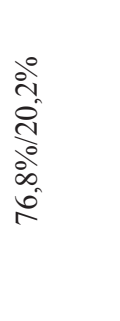 & 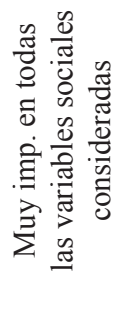 & 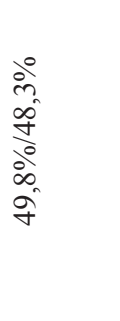 & $\begin{array}{l}\stackrel{0}{2} \\
0 \\
0 \\
\infty \\
\infty \\
\infty\end{array}$ \\
\hline 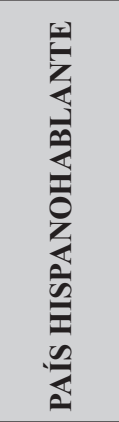 & $\frac{a}{2}$ & 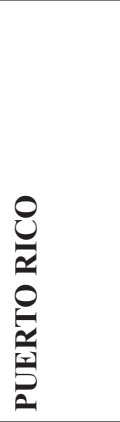 & 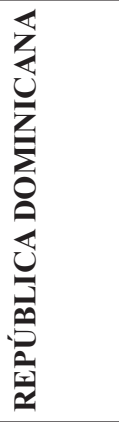 & 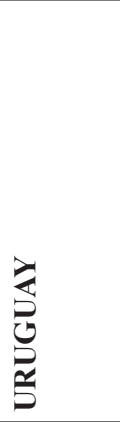 & 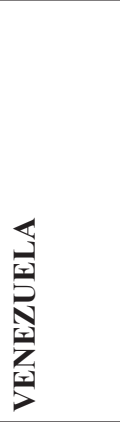 \\
\hline
\end{tabular}

